\title{
Ventricular Approach for Functional Mitral Regurgitation in Cardiomyopathy
}

\author{
Kenji Minakata*, Kazuhiro Yamazaki, Senri Miwa, Masaki Funamoto, Motoyuki Kumagai, \\ Akira Marui, Ryuzo Sakata \\ Department of Cardiovascular Surgery, Kyoto University Graduate School of Medicine, Kyoto, Japan \\ Email: ${ }^{*}$ minakata@kuhp.kyoto-u.ac.jp
}

Received December 19, 2012; revised January 24, 2013; accepted February 5, 2013

\begin{abstract}
Background: The key mechanism of functional mitral regurgitation (FMR) in cardiomyopathy is leaflet tethering caused by displacement of the papillary muscles (PM) due to left ventricular dilatation. The attendant remodeling process is characterized by intraventricular widening between both PM. Recently, surgical ventricular restoration (SVR) has been proposed as a technique to reduce leaflet tethering by improving ventricular geometry. However, it is unknown how SVR improve FMR. Methods and Results: From 2003 to 2010, we surgically treated FMR in 100 patients with idiopathic dilated cardiomyopahy (DCM) or ischemic cardiomyopathy (ICM). Of those, we performed posterior wall exclusion procedures by either resection (the Batista procedure, $n=13)$ or plication $(n=19)$ to approximate papillary muscle distance in a total of 32 patients (DCM in 17, ICM in 15), and these patients formed the cohort of this study. There were two 30-day mortalities (6.3\%). There was no significant change in left ventricular ejection fraction, however, the size of the left ventricle, degree of MR, tethering height and distance of PM significantly decreased after operation and well maintained at the mean follow up of $3.3 \pm 2.1$ years. Conclusions: Posterior wall resection or plication with PM approximation provides excellent reduction of leaflet tethering and MR. Thus, reduction of PM distance may be helpful to treat FMR due to leaflet tethering.
\end{abstract}

Keywords: Cardiomyopathy; Heart Failure Operations; Mitral Regurgitation; Myocardial Remodeling

\section{Introduction}

It is increasingly evident that functional mitral regurgitation (FMR) is more likely to recur or persist after mitral annuloplasty if there is severe mitral leaflet tethering in patients with idiopathic dilated cardiomyopathy (DCM) or ischemic dilated cardiomyopathy (ICM) [1]. Several techniques have been proposed to treat FMR, in addition to the common technique of mitral annuloplasty (MAP) using downsized annuloplasty rings. Most of these techniques aim to reduce leaflet tethering, and are thus referred to as "subvalvular procedures." To date, proposed subvalvular techniques used to treat leaflet tethering include: 1) papillary muscle relocation or approximation [2,3]; 2) chordal cutting [4]; and 3) surgical ventricular restoration (SVR) [5]. Each of these techniques can play an important role in reducing leaflet tethering via different mechanisms. As such, it seems practical to use a combination of these techniques [6,7]. We have aggressively treated patients with FMR using different techniques at our institutions $[8,9]$, and herein we report on the early and mid-term outcomes of our series of patients,

*Corresponding author. focusing mainly on posterior wall exclusion procedures.

\section{Materials and Methods}

\subsection{Patient Selection}

From June 2003 to May 2010, a total of 100 patients who had FMR associated with either ICM $(\mathrm{n}=78)$ or DCM (n $=22$ ) underwent MAP with various types of SVR procedures at our institutions. The indications for SVR procedures included dilated left ventricle (left ventricular end-diastolic dimension $\geq 65 \mathrm{~mm}$ by echocardiography), presence of MR greater than moderate, and presence of congestive heart failure. According to the size and shape of the left ventricle and its myocardial condition, we performed SVR such as overlapping left ventriculoplasty [6] or septal-anterior wall ventricular exclusion (SAVE) with endocardial patch reconstruction [10] not only for patients with ICM due to a previous antero-septal infarcttion, but also for those with DCM. The main purpose of these techniques was to reduce the size and to restore the left ventricle to a more ellipsoidal shape. However, we realized that dilatation of the postero-lateral wall accompanied by an increased distance between both papillary 
muscles may be a more important cause of mitral leaflet tethering. Thus, we started to use a procedure to reduce inter-papillary muscle distance by excluding the posterior wall. During the study period, a total of 32 patients underwent posterior wall exclusion procedures with either resection (the Batista procedure) or plication accompanied with papillary muscle approximation (PMA). These patients formed the cohort of this study. The mean age was $61.7 \pm 12.3$ years $(50 \%$ of patients were at the age $\geq 65$ ), and male gender was predominant (75\%). Preoperatively, all patients underwent coronary angiography, right-side heart catheterization, and myocardial nuclear study whenever feasible. Also, cardiac magnetic resonance study with or without gadolinium enhancement was performed if patients had no permanent pacemakers/defibrillators.

\subsection{Echocardiographic Study}

All patients underwent transthoracic echocardiography before and after operation. Standard two-dimensional and Doppler examination was performed. In addition to the baseline measurements of left ventricular end-diastole dimension (LVEDD) and end-systolic dimension (LVE$\mathrm{SD}$ ), left ventricular volumes (end-diastolic: LVEDV; end-systolic: LVESV) and ejection fraction (EF) were determined by the modified biplane Simpson's method. Degree of mitral regurgitation was assessed quantitatively using proximal isovelocity surface area and vena contracta method [11]. The effective regurgitant orifice (ERO) and regurgitant volume (RV) were calculated by the formula. Then, severity of MR was defined according to the current guideline as none (Grade 0), mild (Grade 1), moderate (Grade 2), moderately severe (Grade 3) and severe (Grade 4). The surgical indication of mitral valve procedures was a presence of MR greater than moderate (ERO $\geq 20 \mathrm{~mm}^{2}$ and/or $\mathrm{RV} \geq 30 \mathrm{ml}$ ). Tethering height was defined as the distance between the plane of the mitral annulus and the coaptation point. This was measured in the mid-systolic parasternal long axis view. The distance between the anterior and posterior papillary muscles was measured with the short-axis view at the level of the papillary muscle insertion to the endocardial surface in end-diastole. Intraoperative transesophageal echocardiography was performed in all the patients to evaluate anatomy, valvular competence and cardiac functions throughout the operation.

\subsection{Operative Technique}

All procedures were conducted on hearts arrested by antegrade tapid crystalloid cardioplegia, followed by tapid blood cardioplegia every 20 - 30 minutes. In addition, retrograde blood cardioplegia may be given in patients with severe coronary artery disease. We do not use terminal hot shot. First, complete coronary revascularization was performed when indicated, and additional antegradecardioplegia was given through saphenous vein grafts which are anastomosed to target coronary arteries in those patients. Then, the first incision was made in the anterior myocardial wall close to the apex parallel to the left anterior descending artery. This incision was extended distally beyond the apex and proximally as needed. The anterior and posterior papillary muscles were carefully identified and mitral leaflets were examined. Usually, the distal incision was further extended towards the posterior wall along to the midline of the anterior and posterior papillary muscles (Figure 1(a)).

As shown in Figure 1(b), our PMA technique was as follows: two or three pledgeted 3 - 0 braided polyester sutures were passed through the body and base of the anterior and posterior papillary muscles, and then these sutures were tied so that both papillary muscles sat side by side. These sutures were usually passed through posterior wall of the left ventricle in between both papillary muscles to plicate the posterior wall as well. When the posterior wall was thin and severely dilated, the incision was extended towards the base of the left ventricle about $2 \mathrm{~cm}$ distal to the coronary sinus, then a small section of the posterior wall was excised as needed (Figure 1(c)). Several deep and wide mattress sutures using 4 - 0 polypropylene sutures supported by felt strips were used to approximate the posterior wall to decrease the interpapillary muscle distance (the Batista procedure; Figure 1(d)). PMA was occasionally performed to secure the alignment of both papillary muscles. The left ventriculotomy was then closed with two layers of sutures.

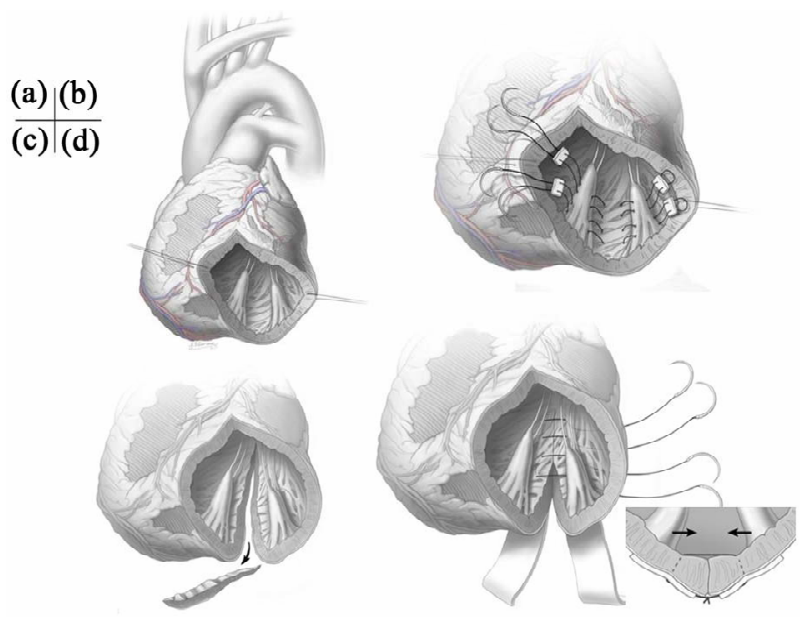

Figure 1. (a) Initial incision of the left ventriculotomy; (b) Papillary muscle approximation with posterior wall plication; (c) The incision is extended posteriorly towards the base of left ventricle; (d) Several deep and wide mattress sutures supported by felt strips are used to approximate the posterior wall to decrease the inter-papillary muscle distance (the Batista procedure). 
MAP was performed in the usual fashion in all patients except one patient who had previously undergone MAP. This patient underwent only our Batista procedure. The rings used for MAP were Carpentier-Edwards Classic rings or Physio rings (Edwards Life Sciences, Irvine, CA, USA). The size used was $26 \mathrm{~mm}(\mathrm{n}=15)$ or $28 \mathrm{~mm}(\mathrm{n}=16)$.

The other additional subvalvular procedure used was chordal cutting. When preoperative echocardiography showed major secondary chordae contributing to the anterior leaflet tethering, an attempt was made to identify these chordae through the left ventriculotomy and then divide them. Care was taken not to cut any strut chordae, which may cause leaflet prolapse.

It has been reported that PMA through a left ventriculotomy per se reduces left ventricular volume to some degree [12]. However, the volume reduction effect of PMA alone may not be enough to achieve adequate ventricular volume reduction. In such cases, additional volume reduction techniques such as overlapping ventriculoplasty [6] or the SAVE procedure [10] were used in addition to PMA, particularly in patients with history of antero-septal myocardial infarction. The technical details of these procedures have been described elsewhere $[6,10]$. In brief, after a ventricular incision was extended proximally along the left anterior descending artery, the left marginal incision was directly sutured to the endocardium of the septal wall using continuous sutures (overlapping ventriculoplasty). Sometimes, an elliptical Dacron patch was sutured to the transitional zone to exclude the akinetic apical-septal area and restore the shape of the ventricle (SAVE procedure). The target left ventricular volume was estimated by the following formula:

$$
\operatorname{LVEDVI}\left(\mathrm{ml} / \mathrm{m}^{2}\right)=1000 \times \text { Cardiac index } / \mathrm{LVEF} \times \mathrm{HR}
$$

$$
\text { (HR : heart rate) }
$$

For example, given the average LVEF of approximately $30 \%$ with heart rate at 90 beats per minute (paced immediately postoperatively), the calculated LVEDVI would be approximately $90-110 \mathrm{ml} / \mathrm{m}^{2}$ under target cardiac index at $2.5-3.0 \mathrm{~L} / \mathrm{min} / \mathrm{m}^{2}$. The sizing device was also sometimes used to estimate the cavity volume. In terms of the selection of additional techniques, the decision was made based on the preexisting myocardial status and the left ventricular size and shape.

\subsection{Data Collection and Statistical Analysis}

Demographic and other patient-related data were obtained from medical records. All patients were contacted during a subsequent outpatient clinic visit for follow-up examination. Echocardiographic studies were routinely performed every $6-12$ months at our institutions or the outpatient clinics of referring physicians. Results are expressed as mean \pm standard deviation. For comparison of each variable between before and after operation, the Wilcoxon test was used and a $p<0.05$ was considered statistically significant. The probability of survival was estimated by the Kaplan-Meier method. All the statistical analyses were performed using the JMP 8.0 software package (SAS, Carey, North Carolina, USA). This study was approved by the Institutional Review Board, and patients and families gave informed consent.

\section{Results}

In this study, surgical techniques used were posterior wall resection (the Batista procedure; $n=13: 8(47 \%)$ in DCM patients and $5(33 \%)$ in ICM patients) or posterior wall plication $(\mathrm{n}=19: 9(53 \%)$ in DCM patients and 10 $(67 \%)$ in ICM patients). PMA was performed in majority of patients $(\mathrm{n}=24: 12(71 \%)$ in DCM patients and 12 $(80 \%)$ in ICM patients) during the posterior wall exclusion. Additional volume reduction techniques used were SAVE procedure $(\mathrm{n}=12)$ and overlapping left ventriculoplasty $(n=5)$. Also, chordal cutting was added in some cases $(n=19)$. Preoperatively, all the patients except one were in New York Heart Association functional class III $(\mathrm{n}=16)$ or IV $(\mathrm{n}=15)$, six patients were inotrope dependent, three patients were in cardiogenic shock, and five patients required IABP support. Other preoperative co-morbidities included chronic renal dysfunction (serum creatinine $\geq 1.5 \mathrm{mg} / \mathrm{dl}, \mathrm{n}=9,28 \%$; and 2 patients were on hemodialysis) and liver dysfunction (serum total bilirubin $\geq 1.5 \mathrm{mg} / \mathrm{dl}, \mathrm{n}=8,25 \%$ ). Concomitant procedures included coronary artery bypass grafting (CABG) in 16 , tricuspid annuloplasy in 10 , maze procedure in 9 and aortic valve replacement in 2 .

The mean aortic cross-clamp time and cardiopulmonary bypass time were $132 \pm 33$ minutes and $194 \pm 38$ minutes, respectively. A total of 15 patients required IABP support at the time of weaning from cardiopulmonary bypass. All the patients could be weaned off without ventricular assist devices. Two patients died within 30 days of surgery $(6.3 \%)$, one from sepsis and the other from low output syndrome associated with refractory supraventricular tachyarrhythmia. Another 2 patients died during hospitalization; one from pneumonia, and the other from multiorgan failure due to congestive heart failure. The overall hospital mortality was $12.5 \%$. As shown in the Table 1, there was no significant improvement in EF, however LVEDD decreased from $65 \pm$ $8 \mathrm{~mm}$ to $61 \pm 7 \mathrm{~mm}(\mathrm{p}<0.001)$, and LVEDVI decreased from $134 \pm 35 \mathrm{ml} / \mathrm{m}^{2}$ to $99 \pm 28 \mathrm{ml} / \mathrm{m}^{2}(\mathrm{p}<0.001)$. There was a significant reduction in degree of MR (from $3.1 \pm$ 1.0 to $0.3 \pm 0.8, \mathrm{p}<0.001)$. Moreover, the tethering height and papillary muscle distance in diastole decreased from $9.4 \pm 2.1 \mathrm{~mm}$ to $2.9 \pm 2.4 \mathrm{~mm}(\mathrm{p}<0.01)$, and $35 \pm 7 \mathrm{~mm}$ to $19 \pm 6 \mathrm{~mm}(\mathrm{p}<0.01)$, respectively. The restricted motion of the anterior leaflet was im- 
Table 1. Echocardiographic data.

\begin{tabular}{|c|c|c|c|}
\hline \multirow{2}{*}{ Variables } & \multirow{2}{*}{ Preoperative $(\mathrm{n}=32)$} & \multicolumn{2}{|c|}{ Postoperative } \\
\hline & & Early $(\mathrm{n}=30)$ & Mid-term $(n=26)$ \\
\hline Left ventricular ejection fraction (\%) & $28 \pm 8$ & $31 \pm 8$ & $31 \pm 8$ \\
\hline Left ventricular end-diastolic dimension (mm) & $65 \pm 8$ & $61 \pm 7^{*}$ & $60 \pm 7^{*}$ \\
\hline Left ventricular end-systolic dimension (mm) & $57 \pm 10$ & $51 \pm 9^{*}$ & $53 \pm 9^{4}$ \\
\hline Left ventricular end-diastolic volume index $\left(\mathrm{ml} / \mathrm{m}^{2}\right)$ & $134 \pm 35$ & $99 \pm 28^{*}$ & $101 \pm 20^{*}$ \\
\hline Left ventricular end-systolic volume index $\left(\mathrm{ml} / \mathrm{m}^{2}\right)$ & $101 \pm 33$ & $72 \pm 28^{*}$ & $80 \pm 27^{\natural}$ \\
\hline Left atrial dimension $(\mathrm{mm})$ & $51 \pm 11$ & $48 \pm 8^{*}$ & $53 \pm 8$ \\
\hline Mitral regurgitation grade & $3.1 \pm 1.0$ & $0.3 \pm 0.8^{*}$ & $0.6 \pm 0.8^{*}$ \\
\hline Tricuspid regurgitation grade & $2.1 \pm 0.9$ & $1.2 \pm 0.8^{\S}$ & $1.5 \pm 0.6$ \\
\hline Estimated right ventricular pressure $(\mathrm{mmHg})$ & $51 \pm 17$ & $42 \pm 14^{\pi}$ & $45 \pm 14$ \\
\hline Tethering height (mm) & $9.4 \pm 2.1$ & $2.9 \pm 2.4^{\pi}$ & $2.0 \pm 1.0^{\curvearrowleft}$ \\
\hline Papillary muscle distance in diastole (mm) & $35 \pm 7$ & $19 \pm 6^{\natural}$ & $20 \pm 8^{\llbracket}$ \\
\hline
\end{tabular}

${ }^{*} \mathrm{p}<0.001$ comparing with preoperative value; ${ }^{9} \mathrm{p}<0.01$ comparing with preoperative value; ${ }^{\S} \mathrm{p}<0.05$ comparing with preoperative value.

proved in almost all the patients. One patient required reoperation due to recurrent severe MR 4 weeks after the operation. The cause of MR was found to be recurrent leaflet tethering presumably due to re-dilatation of the posterior wall. Subsequently, this patient successfully underwent mitral valve replacement and was discharged later.

The mean length of follow-up was $3.3 \pm 2.1$ years. Follow-up echocardiography were performed in 26 patients out of 28 who were discharged home. These studies demonstrated that EF, LVEDD and LVEDVI remained in the same range as when assessed immediate following surgery. However, three patients had developed moderate MR. There were no significant changes in the tethering height and papillary muscle distance at the mid-term follow-up (Table 1). There were 7 late deaths. The cause of late deaths included congestive heart failure in 2, sudden death in 2, sepsis in 2 and cancer in one. Of note, there were no known patients who died of congestive heart failure accelerated by the presence of MR. The cause of congestive heart failure was essentially pump failure due to either systolic or diastolic dysfunction, or combination of these. The 1-, 2- and 3-year survival rates after operation were $78 \%, 68 \%$ and $57 \%$, respectively (Figure 2).

\section{Discussion}

MR is one of the major complications following myocardial infarction, and the presence of MR is associated with excess mortality independent of baseline characteristics

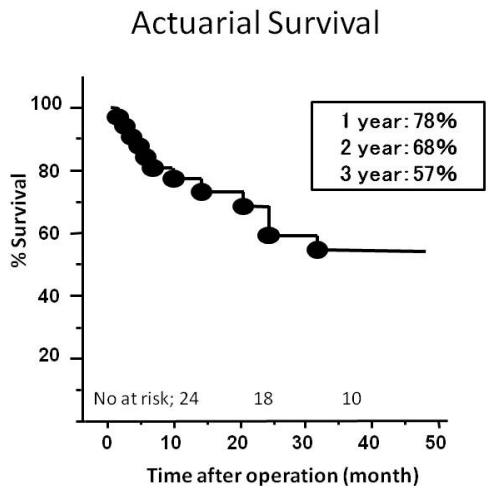

Figure 2. Probability of patient survival after operation.

and degree of ventricular dysfunction [13]. The primary mechanism of FMR is mitral leaflet restriction with tethering due to left ventricular dilatation in addition to some degree of annular enlargement [1]. This is also true in patients with MR due to idiopathic DCM. Bolling et al. have demonstrated that MAP using undersized rings yields excellent early and mid-term outcomes in patients with severe FMR associated with DCM [14]. However, it has been reported that a significant number of patients developed recurrent MR in the early postoperative period [15]. If the leaflet tethering is too severe, the risk of recurrent MR becomes high [1]. In fact, it has been demonstrated that the risk factors for MAP failure include severely dilated left ventricle (LVEDD $\geq 65 \mathrm{~mm}$ ) [16] and severe leaflet tethering (tethering height $\geq 10 \mathrm{~mm}$ ) [17]. In such cases, simple MAP is not sufficient to provide relief. In these circumstances, subvalvular proce- 
dures become necessary to reduce the tethering. To date, several techniques have been proposed to treat leaflet tethering. Liel-Cohen et al. reported that posterior wall plication in an animal model may reduce the leaflet tethering and MR caused by geometric changes after posterior wall myocardial infarction [18]. This technique can be translated to the clinical setting where posterior wall resection (the Batista procedure) may reduce leaflet tethering and FMR as shown in the current study. In fact, Menicanti et al. have demonstrated that intraventricular papillary muscle imbrication at the time of SVR can reduce FMR without ring annuloplasty [19]. Also, Buckberg et al. published a preliminary report showing how papillary muscle dimension can be restored during SVR by PMA [7]. Although the mechanisms of FMR are multifactorial, elongation of papillary muscle distance associated with postero-lateral wall dilatation seems to be one of the most significant determinants. In other words, reducing the inter-papillary muscle distance can be the key component in ameliorating leaflet tethering [19].

Batista's original technique involved a volume reduction achieved by a partial left ventriculectomy in which the posterior wall between anterior and posterior papillary muscles was excised [20]. The essence of this approach was to reduce the wall stress and energy consumption of the myocardium by reducing the radius of the ventricle. A few initial reports showed some favorable outcomes, but a relatively high incidence of early failure and late recurrence of ventricular dilatation precluded its wide clinical application [20]. At the time of these early reports, the issues of MR reduction and leaflet tethering were not discussed at all. Interestingly, McCarthy et al. reported the results of their initial series of the Batista procedure in which repositioning one or both papillary muscles so as to sit side by side, along with MAP, virtually eliminated MR [20].

As an alternative to a left ventricular intervention, Kron et al. reported that direct relocation of the posterior papillary muscle may be an option for patients with minimally dilated left ventricles as a treatment of FMR [2]. Hvass et al. also reported on a technique in which the base of the papillary muscles are drawn together and secured with an intraventricular sling inserted through the left atrium [3]. In addition, it has been reported that cutting the secondary chords contributing to leaflet tethering may improve coaptation and reduce FMR [4]. These techniques may be useful to reduce leaflet tethering, but may not be sufficient to completely eliminate the tethering, particularly in severely dilated hearts.

It is important to evaluate the left ventricular wall characteristics in patients with DCM. The extent of left ventricle (LV) fibrosis and dilatation in DCM is not always homogeneous. One drawback to SVR is that it may compromise viable muscle tissue to restore the LV. The decision of which technique to use must be based on the findings of preoperative myocardial evaluations such as cardiac MRI, nuclear study and echocardiography in addition to direct vision and palpitation. Care should be taken to minimize an excision of viable myocardium to preserve cardiac function. However, as the postero- lateral wall is always impaired without exception in severely dilated hearts with severe FMR in our experiences, the presence of severe FMR due to leaflet tethering per se may be a clinical indicator for the posterior wall resection in SVR.

The ideal surgical treatment for the patients with end-stage heart failure may be heart transplantation. Both the survivorship and the quality of life after heart transplantation have been reported to be excellent. However, it is obvious that heart transplantation is not always an option because of strict indications and limited donor availability. There are a number of factors such as medical condition, psychosocial circumstance and financial issues that restrict transplant candidacy. In the current study, half of the patients were age of 65 or older, and a significant number of patients had had preoperative kidney dysfunction (28\%), liver dysfunction (25\%), inotrope dependent (19\%) and in cardiogenic shock (10\%). Given the severity of preoperative condition and advanced age, the majority of the patients did not meet the candidate criteria for heart transplantation. On the other hand, these patients may benefit from other surgical options such as left ventricular assist devices (LVAD). LVAD implantation using current rotary pumps as a destination therapy may be a good option, especially in elderly patients. According to the latest report from the registry [21], 1- and 2-year survivals after primary LVAD implantation were $74 \%$ and $55 \%$, respectively. The majority of the registry patients could have been sicker than those in the current study, however, the survival after our SVR with MAP procedure in the current study was not inferior to this result from the LVAD registry.

\section{Limitations}

There are several limitations which need to be addressed. First, this is a non-randomized and retrospective study without a control group. Also, this cohort is relatively small and includes heterogeneous subsets of patients. Often, one patient underwent more than two procedures so that it may be difficult to discriminate which procedure contributed more to the reduction in mitral leaflet tethering. Moreover, the mean follow-up period was only 3.3 years. Therefore, further study is needed to determine more clearly the indications for this procedure.

\section{Conclusion}

In patients with severe MR due to leaflet tethering, the posterior wall exclusion procedure by either resection or 
plication, paired with PMA as necessary, provides excellent reduction of leaflet tethering and MR. The reduction of tethering and MR was well maintained at the mid-term follow-up in this series. Thus, reduction of PM distance may be a key component in treating severe FMR.

\section{Acknowledgements}

The authors acknowledge Dr. Tetsuya Ueno and Dr. Yosuke Hisashi for assistance with data collection.

\section{REFERENCES}

[1] E. Kuwahara, Y. Otsuji, Y. Iguro, T. Ueno, F. Zhu, N. Mizukami, et al., "Mechanism of Recurrent/Persistent Ischemic/Functional Mitral Regurgitation in the Chronic Phase after Surgical Annuloplasty: Importance of Augmented Posterior Leaflet Tethering," Circulation, Vol. 114, No. S1, 2006, pp. I529-I534. doi:10.1161/CIRCULATIONAHA.105.000729

[2] I. L. Kron, G. R. Green and J. T. Cope, "Surgical Relocation of the Posterior Papillary Muscle in Chronic Ischemic Mitral Regurgitation," The Annals of Thoracic Surgery, Vol. 74, No. 2, 2002, pp. 600-601. doi:10.1016/S0003-4975(02)03749-9

[3] U. Hvass, M. Tapia, F. Baron, B. Pouzet and A. Shafy, "Papillary Muscle Sling: A New Functional Approach to Mitral Repair in Patients with Ischemic Left Ventricular Dysfunction and Functional Mitral Regurgitation," The Annals of Thoracic Surgery, Vol. 75, No. 3, 2003, pp. 809-811. doi:10.1016/S0003-4975(02)04678-7

[4] E. Messas, J. L. Guerrero, M. D. Handschumacher, C. Conrad, C. M. Chow, S. Sullivan, et al., Chordal Cutting: A New Therapeutic Approach for Ischemic Mitral Regurgitation. Circulation, Vol. 104, 2001, pp. 1958-1963. doi:10.1161/hc4201.097135

[5] M. Di Donato, S. Castelvecchio, J. Brankovic, C. Santambrogio, V. Montericcio and L. Menicanti, "Effectiveness of Surgical Ventricular Restoration in Patients with Dilated Ischemic Cardiomyopathy and Unrepaired Mild Mitral Regurgitation," The Journal of Thoracic and Cardiovascular Surgery, Vol. 134, No. 6, 2007, pp. 1548-1553. doi:10.1016/j.jtcvs.2007.08.031

[6] Y. Matsui, Y. Fukuda, Y. Naito and S. Sasaki, "Integrated Overlapping Ventriculoplasty Combined with Papillary Muscle Plication for Severely Dilated Heart Failure," The Journal of Thoracic and Cardiovascular Surgery, Vol. 127, No. 4, 2004, pp. 1221-1223. doi:10.1016/j.jtcvs.2003.10.044

[7] G. Buckberg, L. Menicanti, S. De Oliveira, T. Isomura and the RESTORE Team, "Restoring Papillary Muscle Dimensions during Restoration in Dilated Hearts," Interactive Cardiovascular and Thoracic Surgery, Vol. 4, No. 5, 2005, pp. 475-477. doi:10.1510/icvts.2005.109868

[8] T. Ueno, R. Sakata, Y. Iguro, H. Yamamoto, M. Ueno, T. Ueno, et al., "Mid-Term Changes of Left Ventricular Geometry and Function after Dor, SAVE, and Overlapping Procedures," European Journal Cardio-Thoracic Surgery, Vol. 32, No. 1, 2007, pp. 52-57. doi:10.1016/j.ejcts.2007.02.030

[9] T. Ueno, R. Sakata, Y. Iguro, H. Yamamoto, M. Ueno, T. Ueno, et al., "Impact of Subvalvular Procedure for Ischemic Mitral Regurgitation on Leaflet Configuration, Mobility, and Recurrence," Circulation Journal, Vol. 72, No. 11, 2008, pp. 1737-1743. doi:10.1253/circj.CJ-08-0449

[10] C. L. Athanasuleas, G. D. Buckberg, A. W. Stanley, W. Siler, V. Dor, M. Di Donato, et al., "Surgical Ventricular Restoration in the Treatment of Congestive Heart Failure Due to Post-Infarction Ventricular Dilation," Journal of the American College of Cardiology, Vol. 44, No. 7. pp. 1439-1445. doi:10.1016/j.jacc.2004.07.017

[11] W. A. Zoghbi, M. Enriquez-Sarano, E. Foster, P. A. Grayburn, C. D. Kraft, R. A. Levine, et al., "Recommendations for Evaluation of the Severity of Native Valvular Regurgitation with Two-Dimensional and Doppler Echocardiography," Journal of the American Society of Echocardiography, Vol. 16, No. 7, 2003, pp. 777-802. doi:10.1016/S0894-7317(03)00335-3

[12] R. U. Nair, S. G. Williams, K. U. Nwafor, A. S. Hall and L. B. Tan, "Left Ventricular Volume Reduction without Ventriculectomy," The Annals of Thoracic Surgery, Vol. 71, No. 6, 2001, pp. 2046-2049. doi:10.1016/S0003-4975(01)02460-2

[13] F. Grigioni, M. Enriquez-Sarano, K. J. Zehr, K. R. Bailey and A. J. Tajik, "Ischemic Mitral Regurgitation: Long-Term Outcome and Prognostic Implications with Quantitative Doppler Assessment," Circulation, Vol. 103, 2001, pp. 1759-1764. doi:10.1161/01.CIR.103.13.1759

[14] S. F. Bolling, F. D. Pagani, G. M. Deeb and D. S. Bach, "Intermediate-Term Outcome of Mitral Reconstruction in Cardiomyopathy," The Journal of Thoracic and Cardiovascular Surgery, Vol. 115, No. 2, 1998, pp. 381-386. doi:10.1016/S0022-5223(98)70282-X

[15] E. C. McGee, A. M. Gillinov, E. H. Blackstone, J. Rajeswaran, G. Cohen, F. Najam, et al., "Recurrent Mitral Regurgitation after Annuloplasty for Functional Ischemic Mitral Regurgitation," The Journal of Thoracic and Cardiovascular Surgery, Vol. 128, No. 6, 2004, pp. 916-924. doi:10.1016/i.jtcvs.2004.07.037

[16] J. Braun, J. J. Bax, M. I. Versteegh, P. G. Voigt, E. R. Holman, R. J. Klautz, et al., "Preoperative Left Ventricular Dimensions Predict Reverse Remodeling Following Restrictive Mitral Annuloplasty in Ischemic Mitral Regurgitation," European Journal of Cardiothoracic Surgery, Vol. 27, No. 5, 2005, pp. 847-853. doi:10.1016/j.ejcts.2004.12.031

[17] A. M. Calafiore, S. Gallina, M. Di Mauro, F. Gaeta, A. L. Iacò, S. D'Alessandro, et al., "Mitral Valve Procedure in Dilated Cardiomyopathy: Repair or Replacement?" The Annals of Thoracic Surgery, Vol. 71, No. 4, 2001, pp. 1146-1152. doi:10.1016/S0003-4975(00)02650-3

[18] N. Liel-Cohen, J. L. Guerrero, Y. Otsuji, M. D. Handschumacher, L. G. Rudski, P. R. Hunziker, et al., "Design of a New Surgical Approach for Ventricular Remodeling to Relieve Ischemic Mitral Regurgitation: Insights from 3-Dimensional Echocardiography," Circulation, Vol. 101, 2000, pp. 2756-2763. doi:10.1161/01.CIR.101.23.2756

[19] L. Menicanti, M. Di Donato, A. Frigiola, G. Buckberg, C. 
Santambrogio, M. Ranucci, et al., "Ischemic Mitral Regurgitation: Intraventricular Papillary Muscle Imbrication without Mitral Ring during Left Ventricular Restoration," The Journal of Thoracic and Cardiovascular Surgery, Vol. 123, No. 6, 2002, pp. 1041-1050. doi: $10.1067 / \mathrm{mtc} .2002 .121677$

[20] P. M. McCarthy, R. C. Starling, J. Wong, G. M. Scalia, T. Buda, R. L. Vargo, et al., "Early Results with Partial Left Ventriculectomy," The Journal of Thoracic and Cardio- vascular Surgery, Vol. 114, No. 5, 1997, pp. 755-763. doi:10.1016/S0022-5223(97)70079-5

[21] J. K. Kirklin, D. C. Naftel, R. L. Kormos, L. W. Stevenson, F. D. Pagani, M. A. Miller, et al., "Second INTERMACS Annual Report: More than 1000 Primary Left Ventricular Assist Device Implants," J Heart Lung Transplant, Vol. 29, No. 1, 2010, pp. 1-10.

doi:10.1016/j.healun.2009.10.009 